\title{
Retraction Note: Quality of life of patients with rheumatoid arthritis in Argentina: reliability, validity, and sensitivity to change of a Spanish version of the Rheumatoid Arthritis Quality of Life questionnaire
}

\author{
Christian A. Waimann • Fernando M. Dal Pra - Maria F. Marengo • \\ Emilce E. Schneeberger • Susana Gagliardi • Jose A. Maldonado Cocco • \\ Mónica Sanchez • A. Garone • Rafael E. Chaparro del Moral • Oscar L. Rillo • \\ Mariana Salcedo • Javier E. Rosa • F. Ceballos $•$ Enrique R. Soriano • Gustavo Citera
}

Published online: 25 October 2013

(C) Clinical Rheumatology 2013

Retraction to: Clin Rheumatol (2012) 31(7):1065 - 1071

DOI 10.1007/s10067-012-1976-6

The Editor has retracted this article due to infringement of copyright. Researchers wishing to use the official RAQoL Spanish translation are requested to contact Galen Research (www.galenresearch.com).

The online version of the original article can be found at http://dx.doi.org/ 10.1007/s10067-012-1976-6.

C. A. Waimann · F. M. Dal Pra • M. F. Marengo •

E. E. Schneeberger - S. Gagliardi - J. A. M. Cocco • M. Sanchez •

M. Salcedo $\cdot$ G. Citera

Instituto de Rehabilitación Psicofísica (IREP) de Buenos Aires,

Buenos Aires, Argentina

A. Garone • R. E. C. del Moral • O. L. Rillo • M. Salcedo

Hospital General de Agudos "Dr. E. Tornú", Buenos Aires,

Argentina

J. E. Rosa $\cdot$ F. Ceballos $\cdot$ E. R. Soriano

Hospital Italiano de Buenos Aires, Buenos Aires, Argentina

G. Citera $(\bowtie)$

Chief Section of Rheumatology, IREP, Echeverría 955,

1428 Buenos Aires, Argentina

e-mail: gustavocitera@gmail.com 